\title{
Psichedelie visioni allucinazioni
}

Sostenibilità/insostenibilità dello sguardo nell'opera di Celati

\section{Ugo Fracassa}

\section{OpenEdition}

\section{Journals}

Edizione digitale

URL: https://journals.openedition.org/cher/733

DOI: $10.4000 /$ cher.733

ISSN: 2803-5992

\section{Editore}

Presses universitaires de Strasbourg

\section{Edizione cartacea}

Data di pubblicazione: 9 juillet 2020

Paginazione: 135-146

ISBN: 979-10-344-0068-3

ISSN: 1968-035X

Notizia bibliografica digitale

Ugo Fracassa, «Psichedelie visioni allucinazioni», reCHERches [Online], 24 | 2020, online dal 20

septembre 2021, consultato il 17 novembre 2021. URL: http://journals.openedition.org/cher/733 ; DOI: https://doi.org/10.4000/cher.733 


\title{
Psichedelie visioni allucinazioni Sostenibilità/insostenibilità dello sguardo nell'opera di Celati
}

\author{
UgO Fracassa ${ }^{1}$
}

$\mathrm{U}$ na modesta proposta per contribuire al lavoro di ricomposizione dei due emisferi poetici nei quali di solito viene scompartita l'opera di Gianni Celati, prima e dopo il novellino padano, può partire da una rivalutazione dell'elemento visivo nel cosiddetto primo tempo della sua narrativa, quello caratterizzato piuttosto dalla verbigerazione e gesticolazione frenetiche della bagarre. A quella stagione appartiene la tetralogia dei romanzi einaudiani, pubblicata tra il 1971 delle irredimibili Comiche, rimaste fuori dal trittico dei Parlamenti buff (1989), e il 1978 del fatidico Lunario del paradiso, libro liminare, travagliato, destinato a servire da soglia verso un'impreveduta maturità. I lavori per la messa in sicurezza dell'opera celatiana, oggi solidamente incasellata, almeno per quanto riguarda l'ala narrativa dell'edificio, tra i volumi dei Meridiani grazie alle cure di Belpoliti e Palmieri, risultano ancora in corso in ambito critico. Ricomporre la varia fenomenologia della scrittura del nostro varrebbe perciò come contributo per una critica sostenibile, quella cioè che consente di dare conto dell'esperienza di scrittura, necessariamente disparata e disomogenea, di un uomo che l'ha praticata nel corso di una vita, senza doversi fingere nella mente un prima e un dopo indipendenti o, peggio, un mostro bicefalo da sacrificare sull'altare della personalità d'autore, del mito della sua supposta integrità. Un importante contributo in questa direzione ha dato recentemente Nunzia Palmieri nel raccogliere alcuni scritti «sulle immagini» nel volume Animazioni e incantamenti (cfr. Celati 2017) e, in particolare, riconoscendo nel sodalizio di Celati col fotografo Carlo Gajani l'avvio di una riflessione sul come del guardare destinata a proseguire, grazie all'incontro coi fotografi del Viaggio in Italia, per culminare negli anni Ottanta in una vera e propria conversione, nel senso appunto di un détournement, di una svolta poetica, nel nome di Luigi Ghirri.

A dire la verità, un paio di letture critiche che risalgono agli anni della svolta sembravano voler fornire per tempo un antidoto atto ad evitare lo scollamento interpretativo che vado deplorando. Da una parte, Guido Almansi su Nuovi

1 Ugo Fracassa, Università Roma Tre. 
argomenti nel 1978, poco prima della pubblicazione del Lunario del paradiso, enfatizzava il quoziente visivo dei tre romanzi editi parlando, in particolare per certi episodi delle Avventure di Guizzardi (1972), di «sceneggiature posteriori al film». Il critico invitava a «non badare troppo a ciò che avviene sotto i nostri occhi sulla superficie grafica del narrato» - la sperimentazione formale e linguistica sotto la quale la scrittura di Celati veniva genericamente rubricata al termine di un decennio segnato dalla neoavanguardia - ma piuttosto a «vedere ciò che avviene sotto gli occhi della nostra mente e della nostra memoria nella evocazione filmico-visiva» (Almansi 1978: 85): un'idea di lettura, insomma, come «cinema naturale» ante litteram. D'altra parte, Guido Fink, scrivendo su Paragone nel 1985, non si peritava di affermare a proposito dei Narratori delle pianure fresco di stampa: "questo libro rifiuta in un certo senso anche le seduzioni oggi imperanti del visivo»; siamo lontani infatti, continuava Fink, dall'ossessione visiva del Calvino di Palomar, libro caratterizzato dalla «onnivora presenza dello Sguardo, da una sorta di totalitarismo, non dissimile in fondo dalla pretesa che valga sempre e comunque un "accordo categorico" fra l'occhio e ciò che esiste» (Fink 1985: 73).

In altre parole, a voler rimettere insieme comiche e pianure, la concitazione della bagarre e la distensione dello sguardo naturale, occorre innanzitutto riconciliare motorio e visivo come i due poli di un unico universo poetico, del resto in gran parte scaturito dal camminar guardando. Si dà il caso che i nostri siano anni favorevoli per un simile impegno esegetico, dal momento che le neuroscienze hanno dimostrato che visivo e motorio sono facoltà co-implicate nei processi corticali di percezione visiva: la visione implica una simulazione incorporata - in particolare vedere qualcuno che afferra un oggetto implica la simulazione di quell'atto con l'attivazione, anche in chi guarda, dei neuroni preposti al motorio (cfr. Rizzolatti e Sinigaglia 2006). A ben guardare, La bottega dei mimi, libro fotografico firmato da Celati insieme a Lino Gabellone, Nicole Fiéloux e Carlo Gajani, sembra fornire, già nel 1977, una vera e propria drammatizzazione del processo mimetico della percezione visiva come descritto nella teoria dei neuroni specchio. Nel libro due compari, identificabili nelle foto di Gajani con lo stesso Celati e col sodale di quei tempi, Lino/Pascal Gabellone, lavorano ad allestire un repertorio di mosse da replicare alla bisogna sul palcoscenico della vita associata.

Si tratta di un espediente di lunga fortuna nella tradizione della slapstick comedy, basato su un comportamento definito mirroring dagli scienziati, basti pensare al gag canonico dello specchio, da Max Linder alla fenomenale versione dei Marx Brothers in Duck's soup (1933). Ne La bottega dei mimi questo gioco di rispecchiamento viene definito "Arte tomatica»:

Poi lavoriamo. Lavoriamo sodo, uno mostra una mossa e l'altro gli fa: «Arte tomatica!» E quello risponde: «Ah sì sì! arte tomatica!» e rifà la mossa ridendo [...] E poi ci copiamo in maniera spudorata l'uno con l'altro. Ma è sempre per via dell'arte tomatica, che quella non l'ha inventata nessuno ma esiste da sola, allora non si riesce a distinguere quello che è mio da quello che è tuo. (Celati 2017: 256) 
Motorio e visivo, insomma, risultano co-implicati fin dal cosiddetto primo tempo dell'arte celatiana e non soltanto a livello creativo, di scrittura letteraria, ma anche a livello di consapevolezza teorica, come dimostra ad esempio questo brano, pressoché coevo, tratto da Alice disambientata: «automatismi mentali o corporei fanno apparire movimenti esterni che condividiamo nella visionarietà»; ad esempio, prosegue, noi «capiamo le mosse di Alice perché corrispondono ad automatismi che potrebbero essere nostri», però «mai chiusi nell'interiorità privata di qualcuno» (Celati 2007: 10).

Il terreno comune alle evoluzioni del corpo comico e dello sguardo naturale nello spazio sarebbe perciò quello di una critica dell'individualità, del mito psicologistico-borghese del soggetto e dell'idolo cartesiano del cogito dal quale deriva. Ciò apparirà con maggiore chiarezza e diffusione discorsiva nella teoresi della visione posteriore alla conversione fotografica nel nome di Ghirri, che rappresenta però solo la pars construens di un discorso iniziato in realtà molti anni prima. Allora, a un'idea di racconto basato sul «sentito dire» corrisponderà, a livello dello sguardo, una poetica del già visto, ispirata alla nozione zavattiniana di «qualsiasità» di ciò che cade sotto l'occhio o sotto l'obiettivo.

L'ipotesi è che accanto alle molte pagine e inquadrature dedicate dal «secondo» Celati a descrivere cosa sia lo sguardo naturale, nei racconti di osservazione o nelle più apertamente filosofiche novelle sulle apparenze o, infine, nei documentari girati tra la via Emilia e il villaggio africano di Diol Kadd, altrettante ve ne siano da ascrivere alla pars destruens di quello stesso discorso sulla visione iniziato già negli anni Settanta. E ciò a partire da una celebre pagina del Lunario del paradiso che propongo di rileggere come parodia (preterintenzionale?) di un celeberrimo ipotesto cinematografico e che rappresenterebbe il manifesto definitivo di visione insostenibile, vero e proprio protocollo di come non si guarda.

Ma andiamo per gradi: al fine di distinguere tra visione sostenibile e insostenibile e prima di portare alcuni esempi dell'una e dell'altra, può intanto risultare utile fare riferimento alla nozione di "percetto» formulata da Celati a proposito dell'antropologo britannico Edward Evan Evans-Pritchard e della sua visione di una palla di fuoco nel cielo di un villaggio azanti. Il «percetto» va inteso come «l'implicito di una visione, figura di qualcosa in un certo momento, all'interno di un certo spazio, all'interno di un modo di vedere e sentire lo spazio esterno. Questo è il vedere figurato della mente non più vero né più illusorio del vedere retinico» ${ }^{2}$ (Celati 2003: 87 ). A ciò si oppone una visione confinata nella

2 Questa idea di «vedere retinico» deve qualcosa alla riflessione di John Berger, autore nel 1972, di un saggio intitolato Ways of seeing: "[Q]uesto vedere che viene prima delle parole, e di cui esse non riescono mai a dare del tutto conto, non dipende dalla reazione meccanica a uno stimolo (la si può vedere in questi termini solo se si isola quell'esigua parte del processo che riguarda la retina) [...] Guardare è un atto 
sua dimensione superficiale, retinica appunto, volta a catturare lo sguardo e a un «afferramento» dell'oggetto di tipo predatorio (in ciò consisterebbe, secondo Celati, la cattiva utopia del realismo-naturalismo, in letteratura e al cinema) ${ }^{3}$. Una simile visione si caratterizza per la nettezza dei contorni, per il nitore dell'immagine - con annesso dispendio tecnologico e di effetti in campo foto e cinemato-grafico - per un'indole prospettica e per una fissità tendenzialmente pornografica, necessariamente compromessa con la logica capitalistica dell'homo oeconomicus. Su riflessioni analoghe Celati tornerà in occasione di uno scritto su Wim Wenders:

[1]a passione di mostrare oggetti di desiderio o di repulsione attraverso un'invadenza descrittiva che li fissa come nudi reperti, in una oggettività fotografica, è propria del realismo o naturalismo: ed è da questi due modi di narrare ottocenteschi che si sviluppa in linea diretta la pornografia. (Celati 2008: 124)

Azzardando un participio sostantivato a partire dall'accezione ottica del termine «risoluzione», si potrebbe ipotizzare come polo negativo del percetto il deverbale risolto, che mi pare abbia anche il vantaggio di alludere all'autoinganno che fonda una simile visione, volta a riconfermare le pretese del cogito individuale, laddove il «percetto» implica «effetti di instabilità», ovvero la particolare condizione in cui l'individuo scopre «che non è sempre lo stesso individuo» (Celati 2003: 81). Pertanto, se predicato del percetto è "qui c'è qualcuno che sta vedendo qualcosa», predicato del risolto sarà un più apodittico: «qui si vede questo».

Venendo agli esempi, propongo di iniziare dal Celati critico, anziché dal narratore. Due personaggi di Federigo Tozzi incarnano infatti con precisione la dicotomia scopica di cui vado dicendo. Nella prefazione a Con gli occhi chiusi il nostro ci presenta Adele, protagonista di un abbozzo di romanzo dello scrittore senese, che, in preda alle allucinazioni, crede di vedere immagini del fuoco nell'acqua di Fontebranda, a Siena:

e questo mostra come il processo di fissazione isterica somigli a quello della visione. La visione non si risolve con un afferramento dell'oggetto, che ci libera dall'inquietudine, ma con una insorgenza emotiva che viene a distrarci da noi stessi - liberando la nostra anima - secondo la formula di Adele. (Celati 1994: XI)

Diversamente da Adele, Domenico, tipico personaggio di benpensante tozziano, pratico, preso nella logica dell'economico, riconduce il mancato accordo fra l'occhio e ciò che esiste - quello che Fink stigmatizzava come «categorico» (1985: 73) - ai sintomi dell'isteria:

di scelta. Il risultato di tale atto è che quanto vediamo si pone alla nostra portata» (Berger 1998: 10-11).

3 Così Celati a proposito della pittura dell'amico Carlo Bertè: «L'effetto dei suoi quadri mi sembra spesso "paradossale", tento di esprimere questo effetto con la parola "naturalezza" per dire che niente è forzato, non vi sono pretese di catturare l'occhio o la mente» (Celati 1985: 6). 
Eccoci tornati alla faccenda dei sintomi isterici: la visione diretta sulle cose, la visione che si risolve in un afferramento meccanico di ciò che cade sotto gli occhi, è cieca ai moti dell'anima. E Domenico è la figura di questa comunissima cecità: la cecità degli occhi aperti, spalancati sul visibile, ma che non vedono niente. (Celati 1994: XVIII)

Eyes wide shut, verrebbe da dire, a proposito dello sguardo del personaggio di Tozzi (ciò che consente di inserire un primo riferimento al cinema di Stanley Kubrick, sul quale tornerò). Quelle che Celati ci presenta sono spesso visioni a cui credere ${ }^{4}$, anche quando indotte chimicamente: è il caso di quelle da chinino, sperimentate in Banda dei sospiri dalla madre di Garibaldi, affetta da febbre spagnola (l'uomo mascherato apparsole finisce per apparire anche al protagonista, ed è lo stesso che aveva salvato la vita allo zio meccanico anni prima!). Come che sia, si è portati a dubitare delle visioni. Pure illusioni ottiche o proiezione dei nostri desideri nel mondo esterno? - si chiede il viaggiatore Astafali in Fata Morgana - fenomeni puramente ottici oppure stati della mente, come credono gli indolenti abitatori di Gamuna Valley? Si tratta in ogni caso, ribadisce Celati, di "percezioni che si caricano di forti intensità affettive o perturbanti, e diventano stati cosiddetti allucinatori. Ma non sono fenomeni molto rari, piuttosto sono continuamente rimossi, perché dipendono da stati troppo intensi della sensibilità» (Celati 2011a: 58-59); e dubitare della loro concretezza si rivela non privo di conseguenze, come dimostra per esempio la novella Il paralitico del deserto, che registra la parabola dell'esistenza dell'infermiere Bugli, da sciupafemmine incallito a eremita, a partire dalla sua stupefazione di fronte a un riflesso sull'acqua: "Ma guarda laggiù nell'acqua, vedi il ponte riflesso? Mettiamo che domani è nuvolo e tu non vedi più il ponte riflesso. Quel ponte lì è una cosa diversa da quando non c'è nessun ponte riflesso nell'acqua?» (Celati 2016: 1293-1294), domanda il personaggio prima di iniziare il proprio percorso di purgazione. Allo stesso modo un «uomo lodevole» scompare, nella quarta novella sulle apparenze, per non aver creduto ai miraggi, in particolare a quelli indotti dalle cromature della moto Yamaha che incantano invece suo figlio ${ }^{5}$. Quest'uomo alieno alle illusioni ottiche crede invece all'esistenza delle merci che vende, per il solo fatto di averne individuato immagine e sigla sul catalogo dei prodotti. Non sorprende allora la sua propensione verso la fissità dell'immagine pornografica: «Ciò che mi spinge è forse l'idea che, esaminando lo sguardo di quelle donne fin troppo nude, [io] possa carpirne l'anima, vedere il significato della sigla»6 . (Celati 2016: 961) La connotazione economica del risolto, come

4 Il riferimento è qui a «Finzioni a cui credere» (cfr. Celati 1984).

5 Non diversamente Ferdinando Amigoni legge come «contrappasso» la sorte toccata al protagonista del racconto Come un fotografo sbarca nel nuovo mondo, fotoreporter "che crede di sapere dove, quando e come si deve guardare», reo di aver degradato lo sguardo a un gioco di forza (Amigoni 2018: 227).

6 Una visione sostenibile discende, secondo Luigi Ghirri, dalla renitenza allo «sguardo onnivoro e un po' pornografico [...] che non sembra lasciare alle cose, ai volti, ai paesaggi la sottile incrinatura di un segreto che ancora possiedono» (Ghirri, 1997: 99). 
propensione verso una visione retinica e come preferenza per l'immagine nitida e fissa, si manifesta inoltre in modo emblematico in queste poche righe tratte dall'edizione del 1989 di Verso la foce. Il brano risulta esemplare perché (caso raro) giustapponendole descrive, nel medesimo tratto discorsivo, le due antitetiche modalità dello sguardo, buona e cattiva visione, percetto e risolto, vincolando il secondo, in clausola, all'immancabile riferimento alla sfera dell'economico:

$\mathrm{Al}$ mattino presto in queste pianure la luce è tutta assorbita dai colori del suolo. C’è un vapore azzurrino che fa svanire le distanze, e oltre un certo raggio si capisce soltanto che le cose sono là, disperse nello spazio. È col sole alto e la luce netta che cominciano a vedersi grandi separazioni, i tagli di luce e ombra fanno apparire forme desolate su tutti i muri, pezzi d'asfalto, siepi o cartelli ai margini d'un movimento generale di traffici e vendite. (Celati 1989b: 78)

È per scongiurare l'incertezza del primo quadro, e le relative Condizioni di luce sulla via Emilia, che un industriale delle scale a chiocciola formula nella sua mente lo sciagurato progetto di un paradiso artificiale. Per allestirlo acquista un appezzamento di terreno:

E un giorno gli era venuta l'idea di costruirvi una grande villa con parco annesso, e di installare nel parco delle turbine per la produzione di venti. Tali turbine avrebbero dovuto spazzare l'aria rendendo le cose esterne sempre nitide e immobili, non più traballanti. (Celati 2016: 918-919)

Nel medesimo racconto è però la vicenda del Menini «dipintore di insegne» a fornire l'exemplum di quanto mortifero possa rivelarsi il perseguimento della risoluzione estrema, del nitore definitivo. Menini infatti verrà ritrovato cadavere, giunto all'ultima tappa della sua insensata quête sensoriale:

Emanuele Menini è stato trovato morto [...] nei pressi di una cabina telefonica. Da quella stessa cabina telefonica Menini aveva poco prima chiamato l'industriale delle scale a chiocciola, per parlargli di qualcosa che aveva visto. Aveva detto d'aver visto una palazzina nelle campagne lì vicino, e d'essere riuscito ad osservarla bene perché l'aria era molto limpida dopo la nevicata, e la neve lasciava spuntare bene i contorni delle cose. (Celati 2016: 917)

Il narratore si recherà infine sul luogo per sperimentare di persona la fatale visione della «palazzina geometrile». Questo il referto stilato: «L'aria era pulita, l'ombra pomeridiana cadeva esattamente tra i due piccoli cipressi che inquadrano la porta, richiamando l'effetto di un luogo perennemente indisturbato che danno i viali dei cimiteri» (Celati 2016: 920).

Un precedente diretto ed altrettanto folle del progetto escogitato dall'industriale padano era, nel Lunario del paradiso, la fantasmagorica luminaria allestita, non senza un certo ingegno artigianale, nel casotto della sua residenza ad Amburgo dal sergente Schumacher. Del rappresentante e venditore di lampadine, nonché ex nazista, è ospite Giovanni (detto Ciofanni), studente italiano squattrinato, errabondo e svagatamente infatuato della di lui figlia Antje. 
Il capitolo trentesimo del romanzo - già ventiduesimo nell'edizione einaudiana del 1978, in seguito a più riprese rimaneggiato a partire dalla pubblicazione nella trilogia del 1989 - ci mostra, in tutto il suo splendore, il paradiso posticcio allestito nel casotto:

Questa sera grande spettacolo del capitano del paradiso e le sue lampadine, ore nove precise visioni celestiali dell'altro mondo, con tutti gli splendori e colori della luce che viaggia alla velocità del pensiero, incluse apparizioni di lucciole e forme geometriche in espansione e le fantasmagorie più meravigliose di questa meravigliosa epoca dello sviluppo umano ${ }^{7}$. (Celati 2016: 648)

Splendore è parola che Celati utilizza, prima che nel Lunario, nella nota scritta per il teatro di Giuliano Scabia nel 1973. Anche in quella sede la parola è declinata in accezione negativa, «come qualcosa che viene giù dall'alto, ma solo nella forma del fasto e della ricchezza, come possibilità di consumo illimitato», a significare «l'abbaglio della rappresentazione» vòlto a «tenere in soggezione un pubblico a disagio» (Celati 1973: 157). E a disagio doveva sentirsi Ciofanni, come Alex il «drugo» - sottoposto in Arancia meccanica (ed eccoci tornati a Kubrick) alla tortura ottica della «cura Ludovico» - intronato su una scomoda seduta in attesa di essere sottoposto alle fantasmagorie dei bulbi luminescenti:

Dopo cena andiamo nel casottino, io e il sergente nazista. Qui devo sedermi su una poltrona da dentista, con schienale ribaltabile, poggiatesta e tutto, in mezzo alla stanza. Che mi voglia cavare un dente per farmi avere le visioni? ${ }^{8}$ (Celati 2016: 649)

L'«abbaglio della rappresentazione», cui Celati si riferiva a proposito del «teatro ufficiale» (Celati 1973: 151-158), si realizza nell'industria cinematografica delle major hollywoodiane col ricorso agli effetti, una volta detti speciali e oggi digitali. Conversando con Massimo Rizzante nel 2005 lo scrittore e documentarista raccontava di aver visto Harry Potter e il Signore degli anelli e di aver riconosciuto in quei film un'idea di fantasia, intesa come «zona torbida della psiche umana da dominare con la razionalità», che definiva tipica del pragmatico mondo anglosassone. Circa il dominio razionale sul fantastico, così concludeva: "Questo compito è ora affidato all'onnipotenza della tecnologia» (Celati 2011a: 70). Così in letteratura come nel cinema, per lo scrittore dei Narratori delle pianure al modello spettacolare del racconto, tipico del romanzo

7 L'antecedente di un simile marchingegno è forse possibile riconoscere nella Dreamachine, citata da William Burroughs (cfr. Kerekes 2003: 5-8), che il pittore Brian Gysin aveva brevettato nel 1961; in quel caso, il lampeggiamento prodotto grazie ad una comune lampadina, sospesa al centro di un cilindro perforato rotante su un giradischi, era in grado di produrre visioni oniriche in quanto sincronizzato sul cosiddetto ritmo alfa, quello delle oscillazioni elettriche emesse da un cervello in stato di profondo rilassamento.

8 Cfr: «Where I was wheeled to, brothers, was like no sinny I had ever viddied before. True enough, one wall was all covered with silver screen $[\ldots]$ and in the middle of the floor facing the screen was like a dentist's chair with all lengths of wire running from it, and I had to like crawl from the wheelchair to this» (Burgess 1962: 111, corsivo mio). 
e del cinema industriali, se ne oppone uno ispirato alla visione naturale, capace di presentarci «il mondo esterno come fenomeno, come serie di apparenze, così come ci appare nelle percezioni quotidiane qualsiasi» (Celati 2011b: 23). Se il riferimento è qui ai maestri del neorealismo, e in particolare al magistero rosselliniano, analoghi esempi Celati è stato in grado di rintracciare nella nostra narrativa primonovecentesca, per esempio nel romanzo Con gli occhi chiusi, per il quale licenziava nel 1994 un'ispirata prefazione:

Caratteristico in Tozzi è l'atto di guardare qualsiasi cosa, e molto spesso particolari casuali e irrilevanti, senza mai la necessità di spiegare e giustificare la cosa vista. (Celati 1994: IX)

Con un considerevole anticipo rispetto a simili riflessioni sui temi del guardare, accumulate a partire dalla seconda metà degli anni Ottanta, il capitolo licenziato come XXII per la prima edizione del Lunario del paradiso può essere letto oggi come contundente parodia della spettacolarizzazione visiva indotta mediante l'uso della tecnologia. Poiché una simile chiave di lettura si rende disponibile previo riconoscimento della fonte filmica sottesa all'episodio, sarà bene ripartire da alcune note di argomento cinematografico. Mentre in Italia, secondo Celati, è proprio con Rossellini che «la spettacolarità del cinema si rivela meno emozionante della visione naturale» (2011a: 58), è negli Stati Uniti che si coglie «un aspetto ricorrente dagli anni Trenta fino a Steven Spielberg: in questo cinema non esiste la possibilità d'uno sguardo su cose qualsiasi» (Celati 2011b: 22). Nell'assenza di occorrenze del nome di Stanley Kubrick nelle interviste, saggi e articoli dedicati da Celati al cinema, questa citazione rappresenta la traccia che ci porta più vicini al regista newyorkese, dal momento che Spielberg è stato suo collaboratore in un progetto cinematografico sull'intelligenza artificiale, portato a termine dopo la scomparsa del regista di 2001: Odissea nello spazio, proprio nel 2001. Sebbene i gusti cinematografici del nostro vadano in direzione opposta rispetto ad una linea kubrickiana, quasi unanimemente riconosciuta come portante nella storia della settima arte del secondo Novecento, la mancata menzione del suo nome da parte dello scrittore, gran conversatore anche in materia di film, non può passare inosservata. Tanto più che tra il 1968 ed il 1970, per il futuro autore di Comiche, all'epoca beneficiario di una borsa di studio a Londra, la sala cinematografica era luogo elettivo, come e più della biblioteca del British Museum: "Questi libri mi sembravano irresistibili, quasi come il cinema, che allora era per me una droga quotidiana»' (Celati 1998: 313). Il citato 2001: Odissea nello spazio fa la sua apparizione sugli schermi, prima statunitensi ed inglesi e poi italiani, proprio nel 1968. Forse una tra le più

9 Vale forse la pena di ricordare che il saggio di Susan Sontag Against interpretation, nel quale si professava predilezione per il cinema rispetto alla letteratura, con toni che sembrano anticipare quelli del Celati avversatore della critica, era uscito nel 1966 (e tradotto in Italia l'anno seguente): «Nei buoni film c'è sempre un'immediatezza che ci libera totalmente dalla smania di interpretare» (Sontag 1967: 23). 
celebri sequenze del cinema mondiale legate ad effetti speciali è la cosiddetta star gate sequence, per la quale il regista si meritò nel 1969 il relativo Premio Oscar, l'unico attribuito alla pellicola. Nonostante l'indicazione nei titoli di coda: «Effetti fotografici speciali di Stanley Kubrick», per realizzare quelle immagini il regista si affidò ad una tecnologia (lo slit-scan) concepita da Douglas Trumbull a partire dall'osservazione del Mechanical Analogue Computer, brevettato per la Lockheed Aircraft Factory da John Whitney, durante la seconda guerra mondiale, come «sistema fotografico ad alta velocità, capace di registrare (e quindi di previsualizzare) la traiettoria dei missili» (Amaducci 2010: 27). La scena ci presenta David Bowman, a bordo della navicella Discovery che fa rotta su Giove, mentre viene proiettato in un viaggio nello spazio-tempo. Lo spettatore sperimenta in soggettiva, dalla sua poltrona, ciò cui il cosmonauta è sottoposto al posto di comando dell'astronave. Al flusso delle immagini solarizzate si alternano primissimi piani di Dave e dei suoi occhi inondati di luce $^{10}$. A proposito di sostenibilità dello sguardo, oltre alle ascendenze belliche dello slit-scan, si segnala pure il riutilizzo dello schema visivo della sequenza nel finale di Behind the Green Door (1972), film che segnò l'inizio della golden age of porn, grazie anche alla distribuzione a livello nazionale fino ad allora preclusa al cinema di genere ${ }^{11}$. Ebbene, i circa dieci minuti della star gate sequence, spesso paragonata ad un trip lisergico in virtù dell'alto tasso psichedelico dell'impianto visivo, sembrano trovare dieci anni dopo a pagina 109 dell'edizione einaudiana del Lunario una versione letteraria, letterale e pressoché interlineare, oltreché parodistica.

Quadrati verdi dentro altri quadrati verdi dentro quadrati che scappano via lontano in uno spazio enorme che li risucchia e risputa come cerchi rossi dentro altri cerchi rossi che vengono avanti e si spaccano in mille rombi di tutti i colori, perdendosi di qua e di là nello spazio enorme come quello delle stelle. E nello spazio enorme spuntavano scie e onde che andavano su e venivano giù; sinusoidi che salgono su un riflesso rosa e scendono su uno violetto, poi stirandosi in strisce che corrono e adesso si inseguono come matte; dal giallo diventato rosso, inseguito dal blu che lo acchiappa intanto che arriva il verde e li supera, raggiunto dal bianco che va fortissimo e ci resta solo lui a far le onde. (Celati 1978: 109)

Ciò che colpisce intanto di questa tessera eventualmente ecfrastica è il fatto che resti invariata nel corso di decenni attraverso i numerosi rimaneggiamenti cui il libro è andato incontro (cfr. Celati 2016: 652), testimoniati dalla numerazione stessa del capitolo, lievitata dal ventidue al trenta. A voler elucubrare, la

10 Per quanto riguarda il colore, Kubrick si affida alla solarizzazione cromatica, una scoperta fototecnica della metà degli anni Sessanta basata sulla sovraesposizione, la stessa che aveva caratterizzato le iconiche copertine dei Beatles realizzate da Richard Avedon.

11 «Il finale di 2001 sarebbe stranamente coinciso con la sequenza finale di Behind the Green Door, un classico della pornografia girato da Jim e Artie Mitchell nel 1975 [sic] a San Francisco, nel quale vengono schizzate delle gocce di sperma, multi colorate e al rallentatore» (Baxter 1999: 299). 
tenuta della lassa descrittiva parlerebbe a favore della presenza di un ipotesto (visivo), restando immutato il quale, la trascrizione non cambierebbe. Appena fuori dai suoi confini testuali le varianti invece si affollano e cade, dalle righe immediatamente successive, l'esplicito riferimento all'uso di droghe:

poniamo che siate di quelli che le hanno viste queste cose: vi rendete conto che l'acido è una limatura di cervello, l'eroina la pausa della morte, questi qui giochetti da lampadina elettrica. (Celati 1978: 109)

Ma poniamo che ve ne intendiate di queste cose; voi sapete come sono i viaggi fuori di sé, in quale spazio enorme si piomba, e come vengono folate di immagini senza regolamentazione nel farnetico. (Celati 2016: 652)

A proposito della star gate sequence, Michel Chion rileva come, dopo una prima ricezione in chiave scientifico-tecnologica, la dimensione del trip divenne rappresentativa di 2001: di «psychedelic experience» parlò il Time, di «ultimate trip» il Christian Science Monitor, mentre in un'intervista rilasciata a Playboy al regista fu chiesto se facesse uso di LSD ${ }^{12}$. Un'ultima suggestione filmica pare provenire infine dalla minima correzione apportata in coda all'episodio, dove il «quadrato di cielo» (Celati 1978: 110) della notte stellata apparsa al malcapitato nel chiuso del casotto viene inopinatamente sostituito da un più cinematografico «rettangolo» (Celati 2016: 653). Una volta inserita la chiave ermeneutica risulta però difficile non far convergere sullo sfondo fantascientifico di 2001: Odissea nello spazio l'orizzonte distopico dell'immediatamente successivo, e già richiamato, Arancia meccanica (1971), vista la scomoda posizione condivisa dai due eroi, entrambi malamente intronati per sottostare a tormenti visivi:

Adesso non tirava più la carrucola, o forse sì, ma adagio adagio. Mi lasciava una lampadina davanti per un certo tempo, se ben capisco, per farmi effetto beccando le mie onde. [...] Nella poltrona non riuscivo a star fermo; [...] Ma dissolto com'ero, non avevo forza di alzarmi, completamente sgonfio. [...] Sono sprofondato nella poltrona da dentista come uno straccio esausto, col vuoto di tristezza nel cervello. (Celati 2016: 651-654)

Ed ecco allora che, a fronte della mancata menzione del regista in articoli, interviste o conversazioni, il lettore si troverebbe, nel bel mezzo del romanzo del '78, davanti ad un vero e proprio centone kubrickiano, ispirato da una ben rilevata vis polemica contro la forzosa spettacolarizzazione della percezione visiva ai limiti del sostenibile.

$\mathrm{Al}$ di là di ogni «investimento intertestuale» ${ }^{13}$ da parte del commentatore e ben lungi da qualsivoglia intentional fallacy (non è questione qui di appurare

12 Questa la risposta di Kubrick: «la droga non è fatta per l'artista [...] crea uno stato di soddisfazione a priori, uccide lo spirito critico» (Chion 2000: 13-14).

13 Mutuo la nozione di «investimento» da uno studio di Daniele Giglioli dedicato alla critica tematica, e propongo di sostituire mentalmente, ai corsivi presenti nel brano citato di seguito, la parola «intertestuale»: «ciò che orienta e determina l'attenzione tematizzante dei diversi interpreti, pur suscettibile di variare all'infinito, non è 
l'ipotetica volontà citazionale da parte dell'autore, sebbene sia difficile propugnare l'innocenza di certe descrizioni in un cinefilo come il nostro nei pieni anni Settanta), lavorare di sponda sulla cosiddetta star gate sequence permette di collegare un datato episodio narrativo - il trip cui veniva sottoposto il protagonista del Lunario del paradiso - con la più recente riflessione di Celati sulla fenomenologia dello sguardo. Procedendo in tal senso non si tarderà a cogliere nell'insostenibile esperienza visiva di Giovanni l'opposto di ciò che l'autore imparerà a riconoscere, grazie soprattutto al sodalizio con Luigi Ghirri, come sguardo naturale. Così facendo sarà infine possibile contribuire a retrodatare la riflessione del nostro sui temi della visione in anticipo sugli anni Ottanta, per riconoscere nel primo Celati il naturale profilo del secondo e, nella denuncia dell'insostenibilità di certe pratiche visive, le avvisaglie di uno sguardo sostenibile ${ }^{14}$.

\section{Bibliografia}

Almansi G., 1978, «Celati uno, due, tre», Nuovi argomenti, 59-60, p. 74-90.

Amaducci A., 2010, Computer grafica. Mondi sintetici e realtà disegnate, Torino, Kaplan.

Amigoni F., 2018, L'ombra della scrittura: racconti fotografici e visionari, Macerata, Quodlibet.

Baxter J., [1999] 2006, Stanley Kubrick: la biografia, Torino, Lindau.

Berger J., 1998, Questione di sguardi, Milano, Il Saggiatore.

Burgess A., [1962] 2012, A Clockwork Orange (Restored Text), New York, W. W. Norton \& Company.

Celati G., 1978, Lunario del paradiso, Torino, Einaudi.

Celati G., 1984, «Finzioni a cui credere», Alfabeta, 67, p. 13-16.

Celati G., 1985, «Pittura come un giardino zen», in Carlo Bertè 1975-1985, Milano, Vallardi, p. 5-7.

Celati G., 1988, «La nostra carne e il suo macellaio. Teatro con visioni, destino, e linguaggio grosso», in Scabia G., Fantastica visione, Milano, Feltrinelli, p. 151-158.

Celati G., 1989a, Parlamenti buffi, Milano, Feltrinelli.

Celati G., 1989b, Verso la foce, Milano, Feltrinelli.

Celati G., 1994, «Prefazione», in Tozzi F., Con gli occhi chiusi; Ricordi di un impiegato, Milano, Feltrinelli, p. VII-XXIV.

affatto arbitrario: l'investimento tematico si radica, infatti, nei più differenti universi estetici ed epistemologici, nei retroterra culturali e sociali, nelle differenze di genere, nelle appartenenze etniche; quanti interpreti, tante domande, e dunque altrettante scommesse» (Giglioli 2001: XIII).

14 Di un simile sguardo rende oggi testimonianza l'attività registica di Gianni Celati. 
Celati G., 1998, «Il progetto Alì Babà, trent'anni dopo», Riga, 14, Alì Babà. Progetto di una rivista 1968-1972, a cura di M. Barenghi e M. Belpoliti, p. 313. Celati G., 2003, «Collezione di spazi», il verri, 21, p. 57-92.

Celati G., 2007, «Sull'epoca di questo libro», in Alice disambientata: materiali collettivi (su Alice) per un manuale di sopravvivenza, a cura di G. Celati, Firenze, Le Lettere, p. 5-11.

Celati G., 2008, "Quando ho visto Nel corso del tempo», Riga, 28, Gianni Celati, a cura di M. Belpoliti e M. Sironi, p. 122-125.

Celati G., 2011a, Conversazioni del vento volatore, Macerata, Quodlibet.

Celati G., 2011b, «Sul cinema italiano del dopoguerra mezzo secolo dopo», in $I l$ cinema di Gianni Celati, a cura di N. Palmieri, Roma, Fandango, p. 19-26.

Celati G., 2016, Romanzi, cronache e racconti, a cura di M. Belpoliti e N. Palmieri, Milano, Mondadori.

Celati G., 2017, Animazioni e incantamenti, con C. Gajani, a cura di N. Palmieri, Roma, L'orma.

Chion M., 2000, Un'odissea del cinema. Il 2001 di Kubrick, Torino, Lindau.

Fink G., 1985, «Da dove vengono tutte le storie», Paragone-letteratura, 426, p. 67-73.

Ghirri L., 1997, Niente di antico sotto il sole. Scritti e immagini per un'autobiografia, a cura di P. Costantini e G. Chiaramonte, Torino, SEI.

Ghirri L., Leone G. e Velati E. (a cura di), 1984, Viaggio in Italia, Alessandria, Il Quadrante.

Giglioli D., 2001, Tema, Scandicci, La nuova Italia.

Kerekes D., 2003, «Dream on», Headpress, 25, William Burroughs \& the Flicker Machine, a cura di D. Kerekes, p. 5-8.

Marrone G., 2005, La Cura Ludovico. Sofferenze e beatitudini di un corpo sociale, Torino, Einaudi.

Rizzolatti G., Sinigaglia C., 2006, So quel che fai: il cervello che agisce e i neuroni specchio, Milano, Raffaello Cortina.

Sontag S., 1967, Contro l'interpretazione, Milano, Mondadori. 